\title{
Therapeutische Notiz
}

$\Lambda$ Vährend es mir melirfach gelungen ist, das diffuse Xant ho ma diabeticoлim durch längere Darreichung von Arson, namentlich auch durch Atoxyl-

meínspritzungen, erheblich zubessern oder zum Schwinden zu bringen, lässt diese Therapie beim persistirenden Xan thorn a palpebrarum im Stich. Da aber bei tlieser zwar nicht bösartigen, doch entstellenden Neubildung alle sonstigen operativen Eingriffe sichtbare Spuren, sogar Ektropien hinterlassen können, so babe ich in letzter Zeit lediglich zur Anwendung der Elektrolyse gegriffen. Die negative Elektrode eines 2 Milliampère starken galvanischen Stromes $\Lambda$ vird in die Neubildung eingeführt und der Strom nach wenigen Minuten unterbrochen, sobald sich eine sichtbare Hyperaemie oder venose Anschwellung der betreffenden Stelle bemerkbar macht. Die Zahl dor Sitzungen richtet sich nach der Grösse und Anzahl der Eruptionen. In einer Sitzung können mehrere Efflorescenzen zur Resorption gebracht werden. Letztere stellt sich nach Ablauf der geringen entzündlichen Reaction im Verlaufe etwa einer Woche ein. Das Verfahren hinterlässt nicht die geringsten Spuren, sodass ein geheilter Fall vom normalen sich in keiner Weise unterscbeidet. Bei etwaigen Wiederwucherungen einzelner, zufällíg stehen geblieboner Punkte kann das Verfahren mit gleichem Erfolge angewandt $\Lambda$ verden. Jedenfalls darf ich die Methode als sicher von nachteiligen Nebenwirkungen frei zur Nachprüfung empfehlen. Auch in mehreren Lehrbüchern finden sich bereits Hinweise auf diesen Gebrauch der Elektrolyse, aber trotzdem scheint derselbe wonig geübt zu werden. Lassar.

Tagresnaehriehten und Persönliches.

Das Komitee für Krebsforschung hat sich entsclilossen, eine Zeit-schrift für Krebsforschung, redigiert von den Herren Prof. Dr. v. Hansemann und Prof. Dr. George Meyer in Berlin, im Yerlage von Gustav Fischer in »lena, herauszugeben, welche einen Sammelpunkt für die wissenschaftlichen Arbeiten auf dem Gebiete der Krebsforschung bilden soil.

Privatdocont Dr. Valentin Sarubin in Charkow ist zum Professor -der Syphilidologie und Dermatologie an der Universität Kasan ernannt wordeo.

Beriehtig·ung.

In dem in diesem liefte enthaltenen Aufsatze: „Schüller, Mitteilung über die protozoenähnlichen Parasiten bei Syphilis” ist auf Seite 335, Zoile 5 von oben hinter „Protoplasmas” zu ergänzen: „der Sporenformen”. 